\title{
ROLE OF OXYGEN REACTIVE SPECIES IN SCHISTOSOMA MANSONI EGG-INDUCED GRANULOMATOUS INFLAMMATION
}

S.W. Chensue, L. Quinlan, G. I. Higashi and S. L. Kunkel

Departments of Pathology and Public Health

The University of Michigan

Ann Arbor, Michigan 48109

Received June 5, 1984

The role of oxygen reactive species in granulomatous hypersensitivity was explored using a model of pulmonary granulomas induced by intravenous injection of eggs from the parasite Schistosoma mansoni. Macrophages from sychronously developing lesions spontaneously released significant quantities of superoxide anion ( 26 nmoles $/ 10^{6} / 2 \mathrm{~h}$ ) by 8 days of development. In contrast, the non-T cell foreign body (Sephadex bead) granuloma macrophages produced only (? nmoles) $10^{6} / 2 \mathrm{~h}$ ) small quantities.

Daily administration of the oxygen scavenger, $\alpha$-tocopherol, by either oral or parenteral routes caused up to $60 \%$ suppression of granuloma size. Moreover, parenteral administration of specific inactivators of $0_{2}^{-}$and $\mathrm{H}_{2} \mathrm{O}$, superoxide dismutase and catalase respectively, resulted in a 36 to $40 \%$ reduction in granuloma size. These data suggest that oxygen reactive species take part in the generation of hypersensitivity granulomas.

Numerous studies have accumulated supporting the role of leukocytegenerated oxygen metabolites in microbial resistance and acute inflammation $(1,2)$. While the bulk of evidence is derived from in vitro experiments, there are several studies that demonstrate an amelioration of tissue destruction and inflammation by employing inhibitors of oxygen metabolites in vivo. For example, parenteral administration of catalase or superoxide dismutase, which respectively catalyze the breakdown of hydrogen peroxide and superoxide anion, have been shown to inhibit immune complex-induced lung damage (3), carrageenan induced footpad edema (4), adjuvant induced arthritis and kaolin-induced granulomas (5).

In a recent report we demonstrated that macrophages isolated from hepatic granulomas formed during experimental murine schistosomasis mansoni spontancously produced significant quantities of superoxide anion during the vigorous unmodulated phase of the disease (6). In the present study, we further characterize the contribution of oxygen metabolites to the generation and maintenance of the hypersensitivity granulomas induced by $\underline{S}$ mansoni eggs. By 
induction of synchronous pulmonary egg granulomas we have demonstrated the time course of $\mathrm{O}_{2}^{-}$production by macrophages isolated from these lesions. Furthermore we show significant inhibition of granuloma size by the in vivo administration of superoxide dismutase or catalase. Likewise, the antioxidant, vitamin E, given orally or parenteraliy resulted in notable inhibition of granuloma formation. These results suggest that oxygen metabolites contribute to the maintenance of hypersensitivity granulomatous reactions.

\section{MATERIALS AND METHODS}

Animals - Female, CBA mice obtained from the Jackson Laboratories (Bar Harbor, Maine) were maintained under pathogen-free conditions and given mouse chow and water ad libitum.

Induction of synchronous pulmonary granulomas and isolated of macrophages. Synchronous pulmonary granulomas were induced by tail vein injection of $3000 \mathrm{~S}$. mansoni eggs. The latter were harvested asepticaliy from the livers mice infected with 200-300 cercariae of the Puerto Rican strain of Schistosoma mansoni as previcusly described (6). The pulmonary lesions were isolated at designated times after embolization and macrophages were collected as previously described (7). Briefly, the granulomatous lungs were excised then chopped in ice cold culture medium with a Waring blender. The granulomas were collected on stainless steel screens then subjected to collagenase digestion (Sigma Chemical Co., St. Louis, MO, Type IV from horseheart) to disperse cells. Macrophages were allowed to adhere overnight then monolayers were washed free of nonadherent cells. Culture medium was RPMI-1640 (Grand Island Biological Co., Grand Island, N.Y.) with $10 \%$ fetal bovine serum (KC Biological, Inc., Lenexa, Kansas) and $10 \mathrm{\mu g} / \mathrm{ml}$ gentamicin (Schering Pharmaceutical Corp., Kene worth, N.J.).

Determination of macrophage superoxide anion production. Superoxide was measured by reduction of ferricytochrome c (Sigma Chemical Co., St. Louis, MO) in Hanks' balanced salts solution as described by johnston et al. (8). A two hour incubation was employed and the total amount of superoxide dismutase inhibitable $0_{2}$ produced was normalized to cell number by direct counting of macrophages adhering to the $35 \mathrm{~mm}$ culture dishes (Corning Glass Works, Corning, N.Y.). Both phorbol myristate acetate $(2 \mu \mathrm{g} / \mathrm{dish})$ and opsonized zymosan (100 $\mu \mathrm{g} / \mathrm{dish}$ ) were used to measure elicited $0_{2}$.

Administration of oxygen metabolite inhibitors. Both catalase and superoxide dismutase (Sigma) were given intraperitoneally at the doses indicated in the text. Vitamin $E, D$ - $\alpha$-tocopherol (Sigma), was given $i . p$. or by feeding tube diluted in peanut oil. Doses of peanut oil provided a control.

Granuloma measurement. Granuloma area was determined by histological examination of tissue sections of lungs harvested at 8 days post egg embolization using an Omicron-Alpha image analyzer (Bausch and Lomb, Rochester, N.Y.) as previously described (9). At least 20 granulomas were measured from each mouse lung with a minimum of five mice per group. The student's t-test was used to compare control and treated groups and $p>.05$ was considered not significant. In general, differences in granuloma size of less than $20 \%$ were not significant.

\section{RESULTS}

\section{Production of superoxide anion by macrophage from synchronously develop-}

ing granulomas. The macrophage is thought to be a key effector cell in the generation and maintenance of granulomas (10). Therefore, we initially wished 


\section{TABLE I}

\begin{tabular}{lcccc}
\hline $\begin{array}{l}\text { Source of Granuloma } \\
\text { Macrophages }\end{array}$ & $\begin{array}{l}\text { Granuloma Area } \\
\left(\times 10^{-3} \mathrm{~m}^{2}\right)\end{array}$ & \multicolumn{2}{c}{$\begin{array}{l}\text { nmoles of } 0_{2}^{-} / 10^{6} \text { cells } / 2 \mathrm{hr} \\
\text { in presence of: }\end{array}$} \\
\hline 4 day egg & $9.6 \pm 0.7$ & $0.0 \pm 0$ & $54.3 \pm 7.8$ & $81.3 \pm 3.3$ \\
8 day egg & $19.7 \pm 1.5$ & $26.2 \pm 5.2$ & $118.4 \pm 16$ & $119.8 \pm 42$ \\
16 day egg & $39.5 \pm 2.2$ & $13.3 \pm 9.2$ & $115.2 \pm 19$ & $149.1 \pm 21$ \\
32 day egg & $22.5 \pm 2.0$ & $13.6 \pm 8.6$ & $96.1 \pm 15$ & $171.5 \pm 16$ \\
8 day Sephadex bead & $7.2 \pm 0.4$ & $2.0 \pm 1.34$ & $105 \pm 16$ & N.D.
\end{tabular}

$\star$

Mean \pm SEM of 4-7 experiments. Each experiment was performed in duplicate or triplicate. N.D. not determined.

to establish the capacity of granuloma macrophages to produce $0_{2}^{-}$and to determine if this could be related to the time course of granuloma development. As shown in Table 1, granuloma macrophages from early 4 d lesions showed no spontaneous $0_{2}^{-}$production. Subsequent to this time spontaneous $0_{2}^{-}$production could be detected, the greatest quantity being observed at 8 days when granuloma development was in its most accelerated phase. In contrast, macrophages from the non-T cel1-mediated Sephadex bead granuloma produced low levels of $0_{2}^{-}$at day 8 . These differences could be largely overcome by providing a maximal stimulus with PMA or zymosan which elicited a large burst of $0_{2}^{-}$from all granuloma macrophages (Table 1). However, macrophages from $4 \mathrm{~d}$ lesions tended to produce lesser amounts.

Effect of $\alpha$-tocopherol on schistosome egg granuloma formation. Vitamin E, $\alpha$-tocopherol, has well known antioxidant activity (11). Thus, it was of interest to determine if this compound would affect granuloma development. Figure 1 shows the effect of daily oral administration of graded doses of Vitamin E. A maximum of $39 \%$ suppression of granuloma formation was obtained with 100 units of oral vitamin $E$ per day. In contrast peanut oil did not significantly inhibit granuloma development. Interestingly, when given by intraperitoneal route, vitamin $E$ caused a $60 \%$ suppression of granuloma formation. 


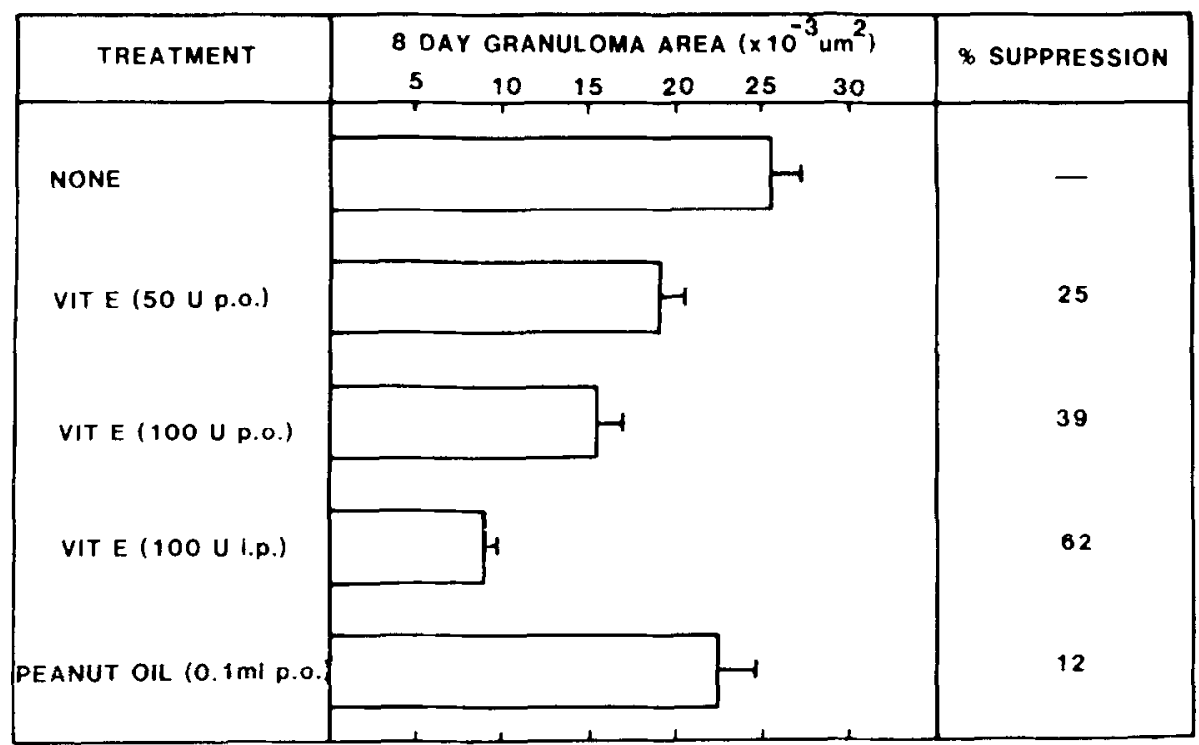

Figure 1. Effect of $\alpha$-tocopherol (vitamin E) on hypersensitivity granuloma formation. Bars represent the mean $+S E$. Five mice per group. A minimum of 20 granulomas were measured from each lung. Mice were given daily $0.1 \mathrm{~m} 7$ doses of vitamin $E$ dissolved in peanut oil by the indicated routes.

\section{Effect of catalase and superoxide dismutase on schistosome egg granuloma}

formation. The above studies suggested that oxygen metabolites may contribute to hypersensitivity granuloma formation. Therefore, we next parenterally administered bovine superoxide dismutase or catalase, specific inactivators of $\mathrm{O}_{2}^{-}$and $\mathrm{H}_{2} \mathrm{O}_{2}$ respectively, to help further identify the metabolites involved. As shown in Figure 2 catalase caused a dose-dependent suppression of granuloma formation. A $30 \%$ suppression was achieved with a $5 \mathrm{mg} /$ day dose. Likewise, SOD caused a $40 \%$ suppression of the 8 day lesion. The control protein, bovine serum albumin did not significantly suppress the lesion at comparable doses. Interestingly, SOD and catalase given in combination did not suppress beyond $40 \%$ (data not shown). These results suggested that both $\mathrm{H}_{2} \mathrm{O}_{2}$ and $\mathrm{O}_{2}^{-}$contribute to the granulomatous inflammatory response.

\section{DISCUSSION}

The capacity of leukocytes to produce a variety of oxygen radicals in vitro is well established, but their precise function in vivo is not fully 


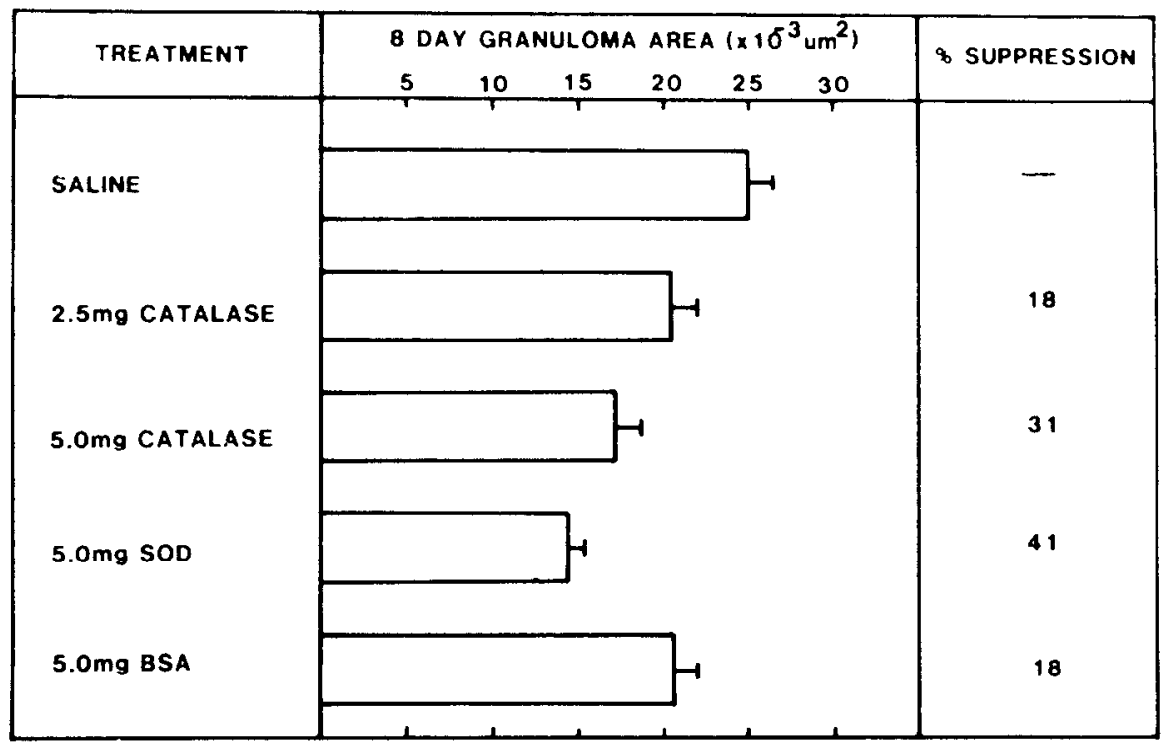

Figure 2. Effect of catalase and superoxide dismutase on hypersensitivity granuloma formation. Bars represent the mean $+S E$. Five mice per group. A minimum of 20 granulomas were measured from each lung. Mice were given daily intraperitoneal injections of the enzymes dissolved in saline.

understood. It is known that rare individuals with genetic defects affecting their ability to generate these products have clearly impaired microbicidal capacity (12). Furthermore, animal studies suggest that oxygen radicals are important in cell and tissue injury $(1,3)$. Attempts to demonstrate the phlogistic activity of oxygen radicals have primarily utilized models of acute inflammation. For example, parenterally administered SOD has been shown to suppress the reversed passive Arthus reaction (13), immune complex-induced alveolitis (3) and pleural effusions (5) or footpad edema (4) induced by carrageenan. However, few studies have implicated a role for oxygen radicals in chronic inflammation.

In a previous report we demonstrated the capacity of macrophages from hepatic granulomas generated in murine schistosomiasis mansoni to spontaneously produce $\mathrm{O}_{2}^{-}$during the vigorous phase of granulomatous hypersensitivity (7). In the present report we employed a model of synchronous schistosome egg-induced pulmonary granuloma formation in order to more specifically delineate the role of oxygen radicals in granulomatous hypersensitivity. Indeed, our results 
confirm and extend our original observation providing evidence that oxygen radicals contribute to granulamatous inflammation. It is noteworthy that significant spontaneous $0_{2}^{-}$release by macrophages was not detected at day 4 . Since this time precedes the accelerated hypersensitivity phase of granuloma formation, this observation may be related to the lack of macrophage activation by immune $T$ cell-derived products. This is further supported by the observed low levels of $\mathrm{O}_{2}^{-}$production by macrophages from Sephadex bead granulomas which lack antigen-specific T-cell involvement. All of the populations produced $\mathrm{O}_{2}^{-}$ with an exogenous stimulus, PMA or zymosan. Thus, regardless of the inciting nidus granuloma macrophages have a higher activation state than resident peritoneal macrophages which fail to produce $\mathrm{O}_{2}^{-}$in response to exogenous stimuli (3).

The ability of vitamin $E$, an oxygen radical scavenger, and oxygen radical degrading enzymes to ameliorate hypersensitivity granuloma formation provides further evidence for a proinflammatory function of reactive oxygen species. The suppressive effect of SOD and catalase implicate both $\mathrm{O}_{2}^{-}$and $\mathrm{H}_{2} \mathrm{O}_{2}$ as participants in granuloma formation. Similar findings were previously reported in a model employing kaolin-induced granulomas (5). The precise mechanism by which reactive oxygen species promote inflammation is not known, but a variety of mechanisms such as local inactivation of neutral proteinases (14), generation of chemotactic factors (13), and iymphocyte activation (15) have been suggested. Interestingly, the release of reactive oxygen species by macrophages has been linked to arachidonic acid metabolism. Specifically, arachidonic acid or its lipoxygenase derivative, 15-HETE, were shown to induce oxygen radical synthesis and may represent second messengers for oxygen metabolism $(16,17)$. This may have relevance to our recent demonstration that schistosome egginduced granuloma macrophage are capable of significant spontaneous arachidonic acid release and metabolism by lipoxygenases (18). Moreover, granuloma formation could be significantly suppressed by agents with anti-lipoxygenase activity. Further analyses are currently underway to delineate the specific biochemical determinants of granulomatous hypersensitivity. 


\section{ACKNOWLEDGEMENT}

This work was supported by NIH grants HL 31237 and HL 31963. The authors wish to express their appreciation for the expert secretarial support of Jeny Breakstone.

\section{REFERENCES}

1. Fantone, J.C., and Ward, P.A. (1982). Am. J. Pathol. 107,397-418.

2. Badwey, J.A., and Karnovsky, M.L. (1980). Ann. Rev. Biochem. 49,695-726.

3. Johnson, K.J., and Ward, P.A. (1981). J. Immunol. 126,2365-2369.

4. Oyangui, Y., (1976). Biochem. Pharmacol. 25,1465-1472.

5. Huber, W., and Saifer, M.G. (1977). in Superoxide and Superoxide Dismutases (Ed. Michelson, A.M., McCord, J.M. and Fridovich, I.) pp. 517-534.

6. Chensue, S.w., Kunkel, S.L., Higashi, G.I., Ward, P.A. and D.L., Boros. (1983). Inf. Immun. 42,1116-1125.

7. Coker, C.M., and VonLichtenberg (1956). Proc. Soc. Exp. Bior. Med. $92,780-782$.

8. Johnston Jr., R.B., Godzik, C.A., and Cohn, Z.A. (1978). J. Exp. Med. $148,115-127$.

9. Chensue, S.W., KunkeT, S.L., Ward, P.A., and Higashi, G. I. (1983). Am. J. Pathol. 111,78-87.

10. Spector, W.G., and Marlano, M. (1975). in Mononuclear Phagocytes in Immunity, Infection and Pathology (ed. Van Furth, R.) pp. 927-942.

11. Dormandy, T.L. (1978). Lancet 1,647-50.

12. Klebanoff, S.J. (1980). Ann. Int. Med. 93,480-489.

13. Petrone, W.F., English, D.K., Wong, K., and McCord, J.M. (1980). Proc. Nat1. Acad. Sci. 77,1159-1163.

14. Matheson, N.R., Wong, D.S., and Travis, J. (1979). Biochem. Biophys. Res. Commun. 88,402-409.

15. Novogrodsky, A., Ravid, A., Rubin, A.L. and Stenze1, K.H. (1982) Proc. Nat1. Acad. Sci. 79,1171-1174.

16. Smith, R.L., and Weidemann, M.J. (1980). Biochem. Biophys. Res. Commun. 97, 973-980.

17. Bromberg, Y.E., Pick, E. (1983) Cell. Immunol. 79,240-252.

18. Kunke1, S.L., Chensue, S.W., and Higashi, G.I. (1984) J. Clin. Invest. (in press). 